\title{
Outcomes and risk factors of patients with acute fatty liver of pregnancy: a multicentre retrospective study
}

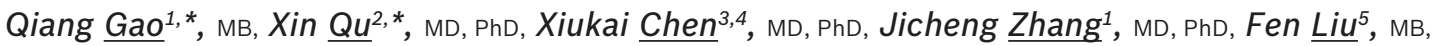 \\ Suochen $\underline{\text { Tian }}^{6}$, MB, MMed, Chunting Wang ${ }^{1}$, MB
}

INTRODUCTION Acute fatty liver of pregnancy (AFLP) frequently causes liver failure in pregnant women. A better understanding of the clinical characteristics, management, outcomes and risk factors of AFLP is required, given its relatively high mortality rate. We aimed to describe the characteristics of AFLP, and further assess its outcomes and potential risk factors from the perspectives of the mother and fetus.

METHODS This was a retrospective cohort study of 133 patients with AFLP hospitalised at four tertiary hospitals in China between January 2009 and April 2014.

RESULTS Among 133 patients, AFLP was diagnosed in the postpartum period for 13 (9.8\%) patients. Potential factors influencing adverse maternal outcome were male fetus $(p=0.04)$, postpartum diagnosis of AFLP $(p<0.01)$, intrauterine fetal death $(p=0.04)$, disseminated intravascular coagulation $(p<0.01)$, prothrombin time $(p<0.01)$ and activated partial thromboplastin time $(p=0.04)$. The frequency of fetal distress $(p=0.03)$ and activated partial thromboplastin time $(p<0.05)$ were significantly higher in pregnancies with dead fetuses than in those where the fetuses survived. Independent risk factors for perinatal maternal mortality were history of legal termination of pregnancy (odds ratio [OR] 1.958, 95\% confidence interval [CI] 1.133-3.385), total bilirubin (OR 1.009, 95\% Cl 1.003-1.014) and serum creatinine (OR 1.010, 95\% Cl 1.003-1.017).

CONCLUSION Compared with total bilirubin and serum creatinine, history of legal termination of pregnancy appeared to be a greater risk factor for maternal mortality among patients with AFLP.

Keywords: fatty liver, liver failure, patient outcome assessment, pregnancy complications, risk factors

\section{INTRODUCTION}

Acute fatty liver of pregnancy (AFLP) is an uncommon, but potentially fatal, disease occurring in the late stage of pregnancy or in the early puerperium with microvesicular fatty infiltration of the liver, which can induce maternal multiorgan failure or even death of the mother and fetus. ${ }^{(1)}$ As early as 1934, AFLP was depicted by Stander and Cadden ${ }^{(2)}$ as 'acute yellow atrophy of the liver'. Existing evidence shows that the aetiology of AFLP may involve oxidative stress on the liver, increased levels of serum arachidonic acid and abnormalities in the intramitochondrial processes of fatty acid oxidation, such as long-chain 3-hydroxyacyl-coenzyme A dehydrogenase deficiencies caused by G1528C mutation. ${ }^{(1,3)}$ The estimated incidence of AFLP ranges from approximately one in 7,000 deliveries to one in 20,000 pregnancies, ${ }^{(2-6)}$ suggesting a low prevalence among pregnant women.

Although the incidence of AFLP is low, it remains a common cause of liver failure in pregnancy and may be accompanied by renal failure, coagulation disorders, hypoglycaemia, encephalopathy and, often, multiple organ dysfunction..$^{(1,7)}$ Both maternal and fetal mortality rates are significantly increased and vary between $1 \%$ and $20 \%$ despite optimal obstetric and medical management. ${ }^{(2,8)}$ With acceptance of the importance of early recognition and diagnosis of AFLP as well as prompt delivery and intensive supportive care, maternal mortality has decreased from as high as $85 \%{ }^{(9)}$ to the current range of $12.5 \%-18 \%$. $^{(10)}$ Simultaneously, neonatal mortality rate has also decreased and is in the range of $7 \%-66 \%$. ${ }^{(3,11)}$ Previous clinical studies on AFLP, largely based on a few patients due to its low prevalence, have found large variations in results vis-à-vis its epidemiology, ${ }^{(2-6)}$ symptoms, ${ }^{(12)}$ complications $^{(12)}$ and outcomes. ${ }^{(3,10,11,13)}$ For instance, Cheng et al ${ }^{(14)}$ found that, besides aetiology, other clinical characteristics of pregnancy (e.g. primiparity, male fetus and multiparous women) that had been identified as potential risk factors in some other studies ${ }^{(9,15)}$ seemed to be partially statistically insignificant in their patients. Cheng et al also indicated that twin pregnancies might be a protective factor for maternal outcome. ${ }^{(14)}$ Conversely, Knight et $\mathrm{al}^{(6)}$ concluded that twin pregnancies were at higher risk of AFLP, similar to Davidson et al, ${ }^{(16)}$ who demonstrated that triplet gestation could increase risk for AFLP.

In view of the insufficient understanding of AFLP and inconsistencies in the related literature, further studies involving larger patient populations are necessary to better guide clinical decision-making, improve prognosis, allow risk stratification and design clinical trials. Our study aimed to analyse a large sample of 133 patients with AFLP in order to: (a) summarise their

\footnotetext{
${ }^{1}$ Department of Critical Care Medicine, Shandong Provincial Hospital Affiliated to Shandong University, Jinan, ${ }^{2}$ Department of Neurosurgery, Xuan Wu Hospital, Capital Medical University, Beijing, China, ${ }^{3}$ Center for Critical Care Nephrology, University of Pittsburgh, School of Medicine, Pittsburgh, USA, ${ }^{4}$ Department of Surgical Intensive Care Unit, Beijing Chao-Yang Hospital, Capital Medical University, Beijing, ${ }^{5}$ Department of Critical Care Medicine, The First Affiliated Hospital of Nanchang University, Nanchang, ${ }^{6}$ Department of Critical Care Medicine, Liaocheng People's Hospital, Liaocheng, China

*These two authors contributed equally as first author in this work.

Correspondence: Dr Chunting Wang, Professor, Department of Critical Care Medicine, Shandong Provincial Hospital Affiliated to Shandong University, 9677 Jing 10 Road, Jinan 250101, China. shengjunshi@126.com
} 
clinical characteristics; and (b) further appraise the outcomes and potential risk factors of maternal and fetal mortality.

\section{METHODS}

We retrospectively analysed a consecutive series of 133 patients with AFLP hospitalised between January 2009 and April 2014 at the departments of critical care medicine or obstetrics at the following hospitals in China: Shandong Provincial Hospital Affiliated to Shandong University, Jinan; The First Affiliated Hospital of Nanchang University, Nanchang; Beijing Chao-Yang Hospital, Beijing; and Liaocheng People's Hospital, Liaocheng. Patients' data was collected from their medical records. The study was approved by the ethics committees at these hospitals.

The diagnosis of AFLP was determined based on both clinical features and laboratory findings, including: (a) symptoms of jaundice, anorexia, fatigue, nausea, vomiting and abnormal liver function during the third trimester of pregnancy; (b) characteristic laboratory findings (e.g. elevated alanine transaminase, bilirubin and serum creatinine levels), leucocytosis, prolonged prothrombin time, reduced fibrinogen and hypoglycaemia; (c) ultrasonography images or computed tomography (CT) examination showing fatty liver; and (d) liver biopsy sample, with characteristic pathological changes, where available. All patients exhibited six or more of the Swansea criteria (Table I), which objectively confirmed the diagnosis of AFLP.(2) During the study period, patients with AFLP for whom liver dysfunction had other causes, such as viral hepatitis, hepatotoxicity, biliary tract disease and cholestasis of pregnancy, were excluded.

The diagnosis of acute liver failure based on clinical and laboratory criteria was made as follows: evidence of coagulopathy, international normalised ratio $\geq 1.5$ or prothrombin activity $\leq 40 \%$; serum total bilirubin $\geq 171 \mu \mathrm{mol} / \mathrm{L}$ or a daily rise $\geq 17.1$ $\mu \mathrm{mol} / \mathrm{L}$; and any degree of mental alteration (encephalopathy) in a patient without pre-existing liver disease and illness $<2$ weeks' duration. ${ }^{(13)}$ Acute renal failure (ARF) was defined as deterioration of renal function over days to weeks and serum creatinine $\geq 265.2$ $\mu \mathrm{mol} / \mathrm{L}$, and acute kidney injury was defined as a deterioration in renal function and serum creatinine $>150 \mu \mathrm{mol} / \mathrm{L} .{ }^{(13)}$

All statistical analyses were performed using IBM SPSS Statistics for Windows version 22.0 (IBM Corp, Armonk, NY, USA). Data was expressed as mean \pm standard deviation, or frequency and percentages. Chi-square test or Fisher's exact test was used for analysis. Logistic regression analysis was performed to analyse relative factors. A p-value $<0.05$ was considered to be statistically significant.

\section{RESULTS}

In total, 140 patients were clinically diagnosed with AFLP during the study period. Among them, five patients were excluded as they did not meet the Swansea criteria and two patients were excluded because of obstetric cholestasis. Eventually, 133 patients with AFLP were enrolled in the study, among whom 13 (9.8\%) were diagnosed in the postpartum period. Abdominal ultrasonography was performed for all patients and $57.1 \%$ had positive results. Biopsy was not done for any patients in view of their serious condition and lack of consent.
Table I. Swansea criteria for the diagnosis of AFLP.

\begin{tabular}{|ll|}
\hline Variable & Finding \\
\hline Vomiting & Positive \\
\hline Abdominal pain & Positive \\
\hline Polydipsia/polyuria & Positive \\
\hline Encephalopathy & Positive \\
\hline Bilirubin & $>14 \mu \mathrm{mol} / \mathrm{L}$ \\
\hline Hypoglycaemia & $<4 \mathrm{mmol} / \mathrm{L}$ \\
\hline Uric acid & $>340 \mu \mathrm{mol} / \mathrm{L}$ \\
\hline Leucocytosis & $>11 \times 10^{6} / \mathrm{L}$ \\
\hline Liver ultrasonography & Ascites or bright \\
\hline AST and ALT & $>42 \mathrm{lU} / \mathrm{L}$ \\
\hline Ammonia & $>47 \mu \mathrm{mol} / \mathrm{L}$ \\
\hline Creatinine & $>150 \mu \mathrm{mol} / \mathrm{L}$ \\
\hline Coagulopathy & $>14 \mathrm{~s}$ \\
\hline PT & $>34 \mathrm{~s}$ \\
\hline APTT & Microvesicular steatosis \\
\hline Liver biopsy & \\
\hline
\end{tabular}

Presence of $\geq 6$ of the Swansea criteria, in the absence of another explanation for liver dysfunction, confirmed the diagnosis of acute fatty liver of pregnancy (AFLP). ALT: alanine transaminase; APTT: activated partial thromboplastin time; AST: aspartate transaminase; PT: prothrombin time

Table II. Demographic characteristics of patients $(n=133)$.

\begin{tabular}{|c|c|}
\hline Variable & No. (\%) \\
\hline Maternal age* (yr) & $27.1 \pm 5.1(17-42)$ \\
\hline Gestational age* (wk) & $36.1 \pm 2.7(21-41)$ \\
\hline $\begin{array}{l}\text { Prodromal symptoms duration } \\
\text { before admission* (day) }\end{array}$ & $10.0 \pm 16.6(0.1-120.0)$ \\
\hline \multicolumn{2}{|l|}{ Treatment } \\
\hline Plasma exchange & $24(18.0)$ \\
\hline Renal replacement therapy & $32(24.1)$ \\
\hline Mechanical ventilation & $43(32.3)$ \\
\hline
\end{tabular}

*Data presented as mean \pm standard deviation (range).

The mean maternal age of the patients was 27.1 years and mean gestational age was 36.1 weeks (Table II). Prodromal symptoms lasted for a mean duration of 10.0 days before admission. The median duration from first symptom to delivery was five days. Detailed maternal manifestations and complications, and main abnormal prenatal laboratory findings are shown in Tables III and IV, respectively. Abnormal blood glucose levels were found in 89 (66.9\%) patients, among whom hypoglycaemia and hyperglycaemia were seen in $76(57.1 \%)$ patients and 13 (9.8\%) patients, respectively. 24 (18.0\%) patients were treated with plasma exchange, $32(24.1 \%)$ patients needed renal replacement therapy and $43(32.3 \%)$ patients required mechanical ventilation. However, no patient underwent liver transplantation. Major reasons for maternal death in our study were multiple organ system failure, disseminated intravascular coagulation and shock.

As shown in Table $\mathrm{V}$, higher proportions of the women who died compared to those who recovered had a history of legal termination of pregnancy (TOP) $(68.2 \%$ vs. $37.8 \%$; $p=0.01)$, 
male fetus $(81.0 \%$ vs. $57.4 \% ; p=0.04)$, AFLP diagnosed in the postpartum period $(31.8 \%$ vs. $5.4 \% ; \mathrm{p}<0.01)$, intrauterine fetal death $(18.2 \%$ vs. $4.5 \%$; $p=0.04)$, disseminated intravascular coagulation $(45.5 \%$ vs. $15.3 \%$; $p<0.01)$, elevated serum creatinine $(86.4 \%$ vs. $55.9 \% ; \mathrm{p}<0.01)$, prolonged prothrombin time $(95.5 \%$ vs. $64.0 \% ; p<0.01)$ and prolonged activated partial thromboplastin time $(95.5 \%$ vs. $74.8 \%$; $p=0.04)$. Similarly, when fetuses were compared in terms of death versus recovery, fetal distress ( $16.7 \%$ vs. $4.8 \% ; p=0.03)$ and prolonged activated partial thromboplastin time $(91.7 \%$ vs. $76.8 \%$; $p<0.05)$ were more common among fetuses that died. Thus, history of TOP, male fetus, postpartum diagnosis of AFLP and intrauterine fetal death might be risk factors for maternal mortality, even as fetal distress was a risk factor for fetal death.

Multivariate logistic regression analysis was carried out for these variables, including gestational age, days from first symptom to delivery, frequency of TOP, gravidity and laboratory findings (Table VI). History of TOP (OR 1.958, 95\% Cl 1.133-3.385), total bilirubin (OR 1.009, 95\% Cl 1.003-1.014) and serum creatinine (OR 1.010, 95\% Cl 1.003-1.017) were independent risk factors for maternal outcome.

\section{DISCUSSION}

Symptoms of AFLP, especially in the early stages of disease, are atypical and can be overlooked. There is disagreement in the published literature regarding the symptoms and clinically common manifestations of AFLP, which included malaise, anorexia, nausea, vomiting, abdominal pain, jaundice, ascites, hypertension and abdominal distension. ${ }^{(4,12-14,17)}$ We found that anorexia, nausea, vomiting and abdominal pain were the main prodromal symptoms, while progressive jaundice and ascites were common signs.

Although the gold standard for diagnosis of AFLP is liver biopsy, this was not done for any patient in our study due to the invasive nature of the procedure, the seriousness of the patients' condition and lack of consent. Much like in our study, the diagnosis of AFLP has seldom been confirmed using liver biopsy in other reports. ${ }^{(6,11,13,14)}$ Also, liver biopsy may not be necessary for the diagnosis of AFLP in most cases, especially for patients with typical clinical findings or severe coagulation disorders. ${ }^{(2,3)}$

Imaging evidence of fatty changes in the liver was commonly demonstrated by abdominal ultrasonography and CT. Knight et al found that classical features of ascites or bright liver were only seen in a quarter of patients who underwent abdominal ultrasonography. ${ }^{(6)}$ All patients underwent ultrasonography in

Table III. Clinical manifestations and complications of patients with acute fatty liver of pregnancy $(n=133)$.

\begin{tabular}{|lc|}
\hline Variable & No. (\%) \\
\hline Manifestation & $67(50.4)$ \\
\hline Anorexia & $63(47.4)$ \\
\hline Jaundice & $60(45.1)$ \\
\hline Ascites & $57(42.9)$ \\
\hline Nausea & $48(36.1)$ \\
\hline Vomiting & $41(30.8)$ \\
\hline Abdominal pain & $31(23.3)$ \\
\hline Abdominal distension & $14(10.5)$ \\
\hline Prenatal vaginal bleeding & \\
\hline Complication & $114(85.7)$ \\
\hline Coagulopathy & $27(20.3)$ \\
\hline Disseminated intravascular coagulation & $109(82.0)$ \\
\hline Hypoproteinaemia & $76(57.1)$ \\
\hline Hypoglycaemia & $74(55.6)$ \\
\hline Acute kidney injury & $13(9.8)$ \\
\hline Acute renal failure & $44(33.1)$ \\
\hline Acute liver failure & $38(28.6)$ \\
\hline Hepatic encephalopathy & $17(12.8)$ \\
\hline Postpartum haemorrhage & $13(9.8)$ \\
\hline Hyperglycaemia & $10(7.5)$ \\
\hline Gastrointestinal bleeding & $9(6.8)$ \\
\hline Multiple organ system failure & $9(6.8)$ \\
\hline Pancreatitis & \\
\hline & \\
\hline
\end{tabular}

Table IV. Prenatal laboratory findings of patients with AFLP $(n=133)$.

\begin{tabular}{|c|c|c|c|c|}
\hline \multirow[t]{2}{*}{ Parameter } & \multirow{2}{*}{$\begin{array}{l}\text { All patients with AFLP } \\
\text { (mean } \pm \text { SD [range]) }\end{array}$} & \multirow[t]{2}{*}{ Normal range } & \multicolumn{2}{|c|}{ Patients with abnormal findings } \\
\hline & & & No. (\%) & Trend \\
\hline Total bilirubin (mmol/L) & $146.6 \pm 102.5(6.9-658.2)$ & $3.4-20.5$ & $124(93.2)$ & Elevated \\
\hline AST (IU/L) & $164.7 \pm 171.1(24.0-852.0)$ & $10.0-40.0$ & $122(91.7)$ & Elevated \\
\hline Total protein $(\mathrm{g} / \mathrm{L})$ & $50.4 \pm 9.1(31.1-75.6)$ & $60.0-80.0$ & $119(89.5)$ & Depressed \\
\hline Albumin (g/L) & $26.1 \pm 5.5(14.0-43.1)$ & $35.0-50.0$ & $107(80.5)$ & Depressed \\
\hline ALT (IU/L) & $162.0 \pm 181.4(13.0-891.0)$ & $10.0-40.0$ & $105(78.9)$ & Elevated \\
\hline APTT (s) & $55.8 \pm 25.6(16.6-149.0)$ & $26.0-36.0$ & $104(78.2)$ & Elevated \\
\hline Leucocytosis $\left(\times 10^{9} / \mathrm{L}\right)$ & $17.3 \pm 7.3(7.8-35.0)$ & $4.0-10.0$ & $103(77.4)$ & Elevated \\
\hline PT (s) & $21.1 \pm 14.9(9.6-120.0)$ & $10.0-14.0$ & $92(69.2)$ & Elevated \\
\hline Glucose (mmol/L) & $4.1 \pm 2.4(0.3-10.3)$ & $3.9-6.1$ & $76(57.1)$ & Depressed \\
\hline Serum creatinine $(\mu \mathrm{mol} / \mathrm{L})$ & $169.3 \pm 93.3(21.3-568.5)$ & $62.0-102.0$ & $81(60.9)$ & Elevated \\
\hline BUN (mmol/L) & $9.3 \pm 5.3(1.3-34.3)$ & $1.7-7.1$ & $65(48.9)$ & Elevated \\
\hline Platelet $\left(\times 10^{9} / \mathrm{L}\right)$ & $122.1 \pm 65.4(12.0-291.0)$ & $100.0-300.0$ & $56(42.1)$ & Depressed \\
\hline
\end{tabular}

AFLP: acute fatty liver of pregnancy; ALT: alanine transaminase; APTT: activated partial thromboplastin time; AST: aspartate transaminase; BUN: blood urea nitrogen; PT: prothrombin time 
Table V. Stratification analysis of the influencing factors with maternal and fetal outcomes.

\begin{tabular}{|c|c|c|c|c|c|c|}
\hline \multirow[t]{3}{*}{ Variable } & \multicolumn{3}{|c|}{ Maternal outcome } & \multicolumn{3}{|c|}{ Fetal outcome } \\
\hline & \multicolumn{2}{|c|}{ No. (\%) } & \multirow[t]{2}{*}{ p-value* } & \multicolumn{2}{|c|}{ No. (\%) } & \multirow[t]{2}{*}{ p-value* } \\
\hline & Recovery & Death & & Recovery & Death & \\
\hline Total & $111(83.5)$ & $22(16.5)$ & & $125(77.6)$ & $36(22.4)$ & \\
\hline Gestational age (wk) & & & 0.78 & & & 0.12 \\
\hline$\leq 36$ & $57(51.4)$ & $12(54.5)$ & & $65(52.0)$ & $24(66.7)$ & \\
\hline$>36$ & $54(48.6)$ & $10(45.5)$ & & $60(48.0)$ & $12(33.3)$ & \\
\hline Time from 1 st symptom to delivery (day) & & & 0.15 & & & 0.11 \\
\hline$\leq 5$ & $64(57.7)$ & $9(40.9)$ & & $71(56.8)$ & $15(41.7)$ & \\
\hline$>5$ & $47(42.3)$ & $13(59.1)$ & & $54(43.2)$ & $21(58.3)$ & \\
\hline History of legal TOP & & & 0.01 & & & 0.21 \\
\hline No & $69(62.2)$ & $7(31.8)$ & & 77 (61.6) & $18(50.0)$ & \\
\hline Yes & $42(37.8)$ & $15(68.2)$ & & $48(38.4)$ & $18(50.0)$ & \\
\hline Gravidity & & & 0.24 & & & 0.16 \\
\hline 1 & $52(46.8)$ & $6(27.3)$ & & $53(42.4)$ & $10(27.8)$ & \\
\hline 2 & $29(26.1)$ & $8(36.4)$ & & $36(28.8)$ & $16(44.4)$ & \\
\hline$\geq 3$ & $30(27.0)$ & $8(36.4)$ & & $36(28.8)$ & $10(27.8)$ & \\
\hline Parity & & & 0.38 & & & 0.08 \\
\hline Primipara & $81(73.0)$ & $14(63.6)$ & & $89(71.2)$ & $20(55.6)$ & \\
\hline Multipara & $30(27.0)$ & $8(36.4)$ & & $36(28.8)$ & $16(44.4)$ & \\
\hline No. of fetuses in pregnancy & & & 0.41 & & & 0.15 \\
\hline 1 & $88(79.3)$ & $20(90.9)$ & & $79(63.2)$ & $29(80.6)$ & \\
\hline 2 & $20(18.0)$ & $2(9.1)$ & & $38(30.4)$ & $6(16.7)$ & \\
\hline 3 & $3(2.7)$ & $0(0)$ & & $8(6.4)$ & $1(2.8)$ & \\
\hline Fetal gender $^{\dagger}$ & & & 0.04 & & & 0.27 \\
\hline Male & $58(57.4)$ & $17(81.0)$ & & $74(59.2)$ & $25(69.4)$ & \\
\hline Female & $43(42.6)$ & $4(19.0)$ & & $51(40.8)$ & $11(30.6)$ & \\
\hline Delivery mode ${ }^{\ddagger}$ & & & 0.33 & & & 0.54 \\
\hline Caesarean section & $96(86.5)$ & $17(77.3)$ & & $108(86.4)$ & $21(80.8)$ & \\
\hline Vaginal delivery & $15(13.5)$ & $5(22.7)$ & & $17(13.6)$ & $5(19.2)$ & \\
\hline Diagnostic time & & & $<0.01$ & & & 0.76 \\
\hline Prenatal diagnosis of AFLP & $105(94.6)$ & $15(68.2)$ & & $113(90.4)$ & $32(88.9)$ & \\
\hline Postpartum diagnosis of AFLP & $6(5.4)$ & $7(31.8)$ & & $12(9.6)$ & $4(11.1)$ & \\
\hline Intrauterine fetal death & & & 0.04 & & & - \\
\hline No & $106(95.5)$ & $18(81.8)$ & & - & - & \\
\hline Yes & $5(4.5)$ & $4(18.2)$ & & - & - & \\
\hline Fetal distress & & & 0.33 & & & 0.03 \\
\hline No & $106(95.5)$ & $20(90.9)$ & & $119(95.2)$ & $30(83.3)$ & \\
\hline Yes & $5(4.5)$ & $2(9.1)$ & & $6(4.8)$ & $6(16.7)$ & \\
\hline Hepatic encephalopathy & & & 0.51 & & & 0.23 \\
\hline No & $78(70.3)$ & $17(77.3)$ & & $83(66.4)$ & $20(55.6)$ & \\
\hline Yes & $33(29.7)$ & $5(22.7)$ & & $42(33.6)$ & $16(44.4)$ & \\
\hline Disseminated intravascular coagulation & & & $<0.01$ & & & 0.22 \\
\hline No & $94(84.7)$ & $12(54.5)$ & & $102(81.6)$ & $26(72.2)$ & \\
\hline Yes & $17(15.3)$ & $10(45.5)$ & & $23(18.4)$ & $10(27.8)$ & \\
\hline Total bilirubin & & & 0.36 & & & 1.00 \\
\hline Normal & $9(8.1)$ & $0(0)$ & & $10(8.0)$ & $2(5.6)$ & \\
\hline Elevated & 102 (91.9) & $22(100.0)$ & & $115(92.0)$ & $34(94.4)$ & \\
\hline Serum creatinine & & & $<0.01$ & & & 0.90 \\
\hline Normal & $49(44.1)$ & $3(13.6)$ & & $29(23.2)$ & $8(22.2)$ & \\
\hline Elevated & $62(55.9)$ & $19(86.4)$ & & $96(76.8)$ & $28(77.8)$ & \\
\hline
\end{tabular}


Table V. (Contd...)

\begin{tabular}{|c|c|c|c|c|c|c|}
\hline \multirow[t]{3}{*}{ Variable } & \multicolumn{3}{|c|}{ Maternal outcome } & \multicolumn{3}{|c|}{ Fetal outcome } \\
\hline & \multicolumn{2}{|c|}{ No. (\%) } & \multirow[t]{2}{*}{ p-value* } & \multicolumn{2}{|c|}{ No. (\%) } & \multirow[t]{2}{*}{ p-value* } \\
\hline & Recovery & Death & & Recovery & Death & \\
\hline AST & & & 0.69 & & & 0.47 \\
\hline Normal & $10(9.0)$ & $1(4.5)$ & & $8(6.4)$ & $4(11.1)$ & \\
\hline Elevated & $101(91.0)$ & $21(95.5)$ & & $117(93.6)$ & $32(88.9)$ & \\
\hline ALT & & & 0.78 & & & 0.63 \\
\hline Normal & $23(20.7)$ & $5(22.7)$ & & $29(23.2)$ & $7(19.4)$ & \\
\hline Elevated & $88(79.3)$ & $17(77.3)$ & & $96(76.8)$ & $29(80.6)$ & \\
\hline PT & & & $<0.01$ & & & 0.26 \\
\hline Normal & $40(36.0)$ & $1(4.5)$ & & $40(32.0)$ & $8(22.2)$ & \\
\hline Prolonged & $71(64.0)$ & $21(95.5)$ & & $85(68.0)$ & $28(77.8)$ & \\
\hline APTT & & & 0.04 & & & $<0.05$ \\
\hline Normal & $28(25.2)$ & $1(4.5)$ & & $29(23.2)$ & $3(8.3)$ & \\
\hline Prolonged & $83(74.8)$ & $21(95.5)$ & & 96 (76.8) & 33 (91.7) & \\
\hline
\end{tabular}

*Based on chi-square test or Fisher's exact test. $\$ 11$ women who were pregnant with male and female fetuses at the same time were excluded from maternal outcome; among these were 10 recoveries and 1 death. $¥ 10$ fetal death in utero not associated with delivery mode were excluded from fetal outcome. AFLP: acute fatty liver of pregnancy; ALT: alanine transaminase; APTT: activated partial thromboplastin time; AST: aspartate transaminase; PT: prothrombin time; TOP: termination of pregnancy

Table VI. Multivariate logistic regression analysis of factors influencing perinatal maternal mortality.

\begin{tabular}{|lllllll|}
\hline Variable & B & SE & Wald & df & OR (95\% Cl) & p-value \\
\hline History of legal TOP & 0.672 & 0.279 & 5.793 & 1 & $1.958(1.133-3.385)$ & $0.016^{*}$ \\
\hline Total bilirubin & 0.009 & 0.003 & 9.027 & 1 & $1.009(1.003-1.014)$ & $0.003^{*}$ \\
\hline Serum creatinine & 0.010 & 0.004 & 7.476 & 1 & $1.010(1.003-1.017)$ & $0.006^{*}$ \\
\hline Constant & -5.668 & 1.097 & 26.687 & 1 & 0.003 & $<0.001^{*}$ \\
\hline
\end{tabular}

${ }^{*} \mathrm{p}<0.05$ was considered statistically significant. Cl: confidence interval; df: degrees of freedom; OR: odds ratio; SE: standard error; TOP: termination of pregnancy

our study and $57.1 \%$ of patients had positive results. Similar to Knight et al's findings, ${ }^{(6)}$ few patients were diagnosed by CT in our study. Although CT may be considered a better option than ultrasonography when AFLP is suspected in a patient, ultrasonography is used more frequently for pregnant women, as it is difficult for these patients to accept $\mathrm{CT}$ given the possible harmful effects of radiation on the fetus. Wei et al ${ }^{(18)}$ reported that the rates of positive AFLP diagnosis by ultrasonography and $\mathrm{CT}$ were $79.7 \%$ and $85.3 \%$, respectively. However, the difference between the two methods was not statistically significant. In our study, we did not attempt to identify women with suspected AFLP using magnetic resonance imaging because of the undefined role of this modality in the diagnosis of AFLP. ${ }^{(19)}$ However, the latest research has shown that magnetic resonance imaging-based liver fat quantification may be an effective tool to obtain a secure diagnosis of AFLP without liver biopsy. ${ }^{(20)}$

Early diagnosis, immediate delivery and comprehensive supportive treatment are the mainstay for the management of AFLP. The occurrence of complications is often associated with poor outcomes. Similar to earlier studies, ${ }^{(17)}$ the major complications of AFLP observed among our patients were coagulopathy and hypoproteinaemia. Acute liver failure and ARF are the most significant and life-threatening complications of AFLP. ${ }^{(13)}$ Among 133 patients, 74 (55.6\%) patients experienced acute kidney injury, but only 13 (9.8\%) patients developed ARF. Hence, the morbidity of ARF in our study was far less than in other reports. ${ }^{(12,17)}$ Only
$32(43.2 \%)$ out of 74 patients with acute kidney injury in our cohort received renal replacement therapy. It is possible that early intervention, by way of renal replacement therapy, may have checked the further worsening of acute kidney injury in our patients, possibly resulting in the lower incidence of ARF. Ganesan and Maynard found that the cause of ARF in patients with AFLP may be related to early suppression of the $\beta$-oxidation of fats. ${ }^{(21)}$

In our series, 44 (33.1\%) patients developed acute liver failure and $24(18.0 \%)$ patients were treated with plasma exchange. Liver transplantation was not reported in our patients, although it has been suggested by Pereira et al ${ }^{(22)}$ for patients with hepatic encephalopathy, severe metabolic acidosis or worsening coagulopathy. The role of liver transplantation in patients with AFLP is very limited due to the huge potential for recovery after delivery. Xiong et al have suggested that acute liver failure and ARF in patients with AFLP are reversible. ${ }^{(13)}$ Related pathological findings were also reported by Rolfes and Ishak. ${ }^{(23)}$

Some clinical characteristics of pregnancy, such as primiparity, male fetus, multiple pregnancies and experiencing preeclampsia, were previously identified as potential risk factors for AFLP. ${ }^{(6,16,18,24)}$ In our study, we identified history of TOP, male fetus, postpartum diagnosis of AFLP and intrauterine fetal death as the potential risk factors for adverse maternal outcome. Postpartum diagnosis of AFLP, which entails delayed comprehensive management of the condition, may be caused by the absence of early clinical manifestations and/or positive 
laboratory findings. While our data confirms the importance of early diagnosis of AFLP in pregnant woman, the diagnostic time appeared to have no effect on fetal outcome.

In our study, maternal and fetal mortality rates were $16.5 \%$ and $22.4 \%$, respectively. This finding is similar to those reported previously ${ }^{(3,10)}$ and highlights the vulnerability of patients with AFLP. There were 22 (16.5\%) women with twin pregnancies in our study, which was similar to another study. ${ }^{(6)}$ A retrospective study by Cheng et $\mathrm{al}^{(14)}$ found significant differences between patients who survived and those who died with respect to twin pregnancies. The proportion of mothers with twin pregnancies in their study $(28.1 \%)$ was much higher than in ours $(16.5 \%)$ and another previous report (18.0\%). ${ }^{(6)}$ However, it is possible that their finding was incidental and, given the small size of their sample, cannot be generalised to all populations. Having twin pregnancies might be a potential protective factor for maternal outcome, but we did not find any statistical significance in our study.

The difference between fetal survival and death in this study was not statistically significant for women with multiparity, singleton pregnancies, male fetus, vaginal delivery, history of TOP and postpartum diagnosis of AFLP. However, the frequency of fetal distress was significantly higher among dead fetuses than those that survived, such that fetal distress might be a risk factor for fetal mortality $(p=0.03)$. Logistic regression analysis demonstrated that history of TOP, total bilirubin and serum creatinine were independent risk factors for maternal outcome. Importantly, the mortality rate among patients with a history of TOP was nearly 1.958 times that of patients without such history. Related research also shows that prothrombin time and international normalised ratio are risk factors for fatal complications in patients with AFLP, and perinatal mortality is associated with the level of fibrin degradation products. ${ }^{(25)}$

A key strength of our study was that data was sourced from tertiary hospitals and our sample was larger than that of previous reports. To the best of our knowledge, the potential risk factors we identified have not been previously described. Logistic regression analysis identified some independent risk factors of patient outcome, with a history of TOP being associated with worse and adverse maternal outcomes.

Nevertheless, our study was not without limitations. First, we did not evaluate the morbidity of AFLP, due to the deficiency of data on total pregnant women during the study period. Second, we could not explain why a history of TOP was associated with mortality for patients with AFLP. It is possible that this association between a history of TOP and poor maternal outcome in our cohort was a matter of chance and not causative. More extensive studies may be needed to establish or negate the existence of such a relationship. Third, given the retrospective nature of our study, we were unable to determine patient outcomes following discharge from the hospital.

To conclude, the main prodromal symptoms of AFLP were anorexia, nausea, vomiting and abdominal pain, while progressive jaundice and ascites were common signs among our patients. We found that male fetus, postpartum diagnosis of AFLP, intrauterine fetal death, disseminated intravascular coagulation, prolonged prothrombin time and activated partial thromboplastin time were potential risk factors for maternal outcome. Similarly, fetal distress and prolonged activated partial thromboplastin time were risk factors for fetal mortality. History of TOP, total bilirubin and serum creatinine were independent risk factors for maternal mortality.

\section{ACKNOWLEDGEMENT}

This study was funded by the National Natural Science Foundation of China (no. 81372473).

\section{REFERENCES}

1. Hay JE. Liver disease in pregnancy. Hepatology 2008; 47:1067-76.

2. Joshi D, James A, Quaglia A, Westbrook RH, Heneghan MA. Liver disease in pregnancy. Lancet 2010; 375:594-605.

3. Papafragkakis $\mathrm{H}$, Singhal S, Anand S. Acute fatty liver of pregnancy. South Med J 2013; 106:588-93

4. Nelson DB, Yost NP, Cunningham FG. Acute fatty liver of pregnancy: clinical outcomes and expected duration of recovery. Am J Obstet Gynecol 2013; 209:456.e1-7.

5. Vigil-de Gracia P, Montufar-Rueda C. Acute fatty liver of pregnancy: diagnosis, treatment, and outcome based on 35 consecutive cases. J Matern Fetal Neonatal Med 2011; 24:1143-6.

6. Knight M, Nelson-Piercy C, Kurinczuk JJ, Spark P, Brocklehurst P. A prospective national study of acute fatty liver of pregnancy in the UK. Gut 2008; 57:951-6.

7. Lau HH, Chen YY, Huang JP, et al. Acute fatty liver of pregnancy in a Taiwanese tertiary care center: a retrospective review. Taiwan J Obstet Gynecol 2010; 49:156-9.

8. Fesenmeier MF, Coppage KH, Lambers DS, Barton JR, Sibai BM. Acute fatty liver of pregnancy in 3 tertiary care centers. Am J Obstet Gynecol 2005; 192:1416-9.

9. Kaplan MM. Acute fatty liver of pregnancy. N Engl J Med 1985; 313:367-70.

10. Rajasri AG, Srestha R, Mitchell J. Acute fatty liver of pregnancy (AFLP)--an overview. J Obstet Gynaecol 2007; 27:237-40.

11. Meng J, Wang S, Gu Y, et al. Prenatal predictors in postpartum recovery for acute fatty liver of pregnancy: experiences at a tertiary referral center. Arch Gynecol Obstet 2016; 293:1185-91.

12. Minakami $H$, Morikawa $M$, Yamada $T$, et al. Differentiation of acute fatty liver of pregnancy from syndrome of hemolysis, elevated liver enzymes and low platelet counts. J Obstet Gynaecol Res 2014; 40:641-9.

13. Xiong HF, Liu JY, Guo LM, Li XW. Acute fatty liver of pregnancy: over six months follow-up study of twenty-five patients. World J Gastroenterol 2015; 21:1927-31.

14. Cheng $N$, Xiang $T, W u X$, et al. Acute fatty liver of pregnancy: a retrospective study of 32 cases in South China. J Matern-Fetal Neonatal Med 2014; 27:1693-7.

15. Kayem G, Kurinczuk J, Lewis G, et al. Risk factors for progression from severe maternal morbidity to death: a national cohort study. PLoS One 2011; 6:e29077.

16. Davidson KM, Simpson LL, Knox TA, D'Alton ME. Acute fatty liver of pregnancy in triplet gestation. Obstet Gynecol 1998; 91(5 Pt 2):806-8.

17. Zhou G, Zhang X, Ge S. Retrospective analysis of acute fatty liver of pregnancy: twenty-eight cases and discussion of anesthesia. Gynecol Obstet Inves 2013; 76:83-9.

18. Wei Q, Zhang L, Liu X. Clinical diagnosis and treatment of acute fatty liver of pregnancy: a literature review and 11 new cases. J Obstet Gynaecol Res 2010; 36:751-6.

19. Castro MA, Ouzounian JG, Colletti PM, et al. Radiologic studies in acute fatty liver of pregnancy. A review of the literature and 19 new cases. J Reprod Med 1996; 41:839-43.

20. Châtel P, Ronot M, Roux O, et al. Transient excess of liver fat detected by magnetic resonance imaging in women with acute fatty liver of pregnancy. Am J Obstet Gynecol 2016; 214:127-9.

21. Ganesan C, Maynard SE. Acute kidney injury in pregnancy: the thrombotic microangiopathies. J Nephrol 2011; 24:554-63.

22. Pereira SP, O'Donohue J, Wendon J, Williams R. Maternal and perinatal outcome in severe pregnancy-related liver disease. Hepatology 1997; 26:1258-62.

23. Rolfes DB, Ishak KG. Acute fatty liver of pregnancy: a clinicopathologic study of 35 cases. Hepatology 1985; 5:1149-58.

24. Lee NM, Brady CW. Liver disease in pregnancy. World J Gastroenterol 2009; 15:897-906

25. Zhang YP, Kong WQ, Zhou SP, Gong YH, Zhou R. Acute fatty liver of pregnancy: a retrospective analysis of 56 cases. Chin Med J (Engl) 2016; 129:1208-14. 